KYUNGPOOK Math. J. 54(2014), 333-339

http://dx.doi.org/10.5666/KMJ.2014.54.2.333

\title{
On the Omega Limit Sets for Analytic Flows
}

JAEYOO CHOY*

Department of Mathematics, Kyungpook National University, Sankyuk-dong, Bukgu, Daegu 702-701, Republic of Korea

e-mail : choy@knu.ac.kr

HAHNG-YUN CHU ${ }^{\dagger}$

Department of Mathematics, Chungnam National University, 99 Daehak-ro, YuseongGu, Daejeon 305-764, Republic of Korea

e-mail : hychu@cnu.ac.kr

ABstraCt. In this paper, we describe the characterizations of omega limit sets $(=\omega$-limit set) on $\mathbb{R}^{2}$ in detail. For a local real analytic flow $\Phi$ by $z^{\prime}=f(z)$ on $\mathbb{R}^{2}$, we prove the $\omega$-limit set from the basin of a given attractor is in the boundary of the attractor. Using the result of Jiménez-López and Llibre [9], we can completely understand how both the attractors and the $\omega$-limit sets from the basin.

\section{Introduction}

Attractors and $\omega$-limit sets, arising from their ubiquitous applications in Dynamical Systems, have played an important role in the field with the useful properties. Especially, these are used to describe the time behavior for dynamical systems, and to provide the dynamicists with certain notions for localizing the complexity. For a manifold $M$ with a vector field on it, and a point $q \in M$, we denote the integral curve (with the initial point $q$ ) by $Z_{q}(t)$. Let an open interval $\left(a_{q}, b_{q}\right)$, possibly with $a_{q}=-\infty$ or $b_{q}=\infty$, be the maximal domain on which $Z_{q}(t)$ is defined. Let us define the $\omega$-limit set of $q$ by

$$
\omega(q)=\left\{x \in M: x=\lim _{n \rightarrow \infty} Z_{q}\left(t_{n}\right) \text { for some sequence } t_{n} \rightarrow b_{q} \text { as } n \rightarrow \infty\right\} .
$$

In the paper, we restrict our interest on $\mathbb{R}^{2}$. Let $f: \mathbb{R}^{2} \rightarrow \mathbb{R}^{2}$ be a real

* Corresponding Author.

$\dagger$ The first author.

Received February 13, 2014; accepted May 22, 2014.

2010 Mathematics Subject Classification: 37C10, 37C70.

Key words and phrases: attractors, $\omega$-limit sets, analytic flows.

This research was supported by Kyungpook National University Research Fund, 2013.

The second named author was supported by Basic Science Research Program through the National Research Foundation of Korea(NRF) funded by the Ministry of Education, Science and Technology(2010-0013784). 
analytic map. Then $f$ defines a real analytic vector field on $\mathbb{R}^{2}$, i.e., $z(t)^{\prime}=f(z(t))$ gives the associated analytic flow. Define $\Phi: D \rightarrow \mathbb{R}^{2}$ by $\Phi(t, u)=Z_{u}(t)$ where $D \subset(-\infty, \infty) \times \mathbb{R}^{2}$ is the maximal open domain on which the ODE has solution. We say $\Phi$ defined by $z^{\prime}=f(z)$ and $\Phi$, a local real analytic flow.

Jiménez López-Llibre [9] gave the complete list of topologies of $\omega$-limit sets for local real analytic flows. In a system of ordinary differential equations on $\mathbb{R}^{d}$, [13] proved that the boundary of an attractor is the $\omega$-limit set of a boundary of a corresponding attractor block in a compact version. In [8], it is obtained that a local topological and dynamical description of an expansive attractors on compact surfaces. Moreover, it is also proved that the above attractors on compact surfaces can be decomposed into invariant sets, and the attractors are hyperbolic except possibly at a finite number of periodic points.

A direct and natural question from the above listed works, can be posed for the attractors. To be precise, for a prescribed $\omega$-limit set $\Omega$, does there exist an attractor $A$ having $\Omega$ as the boundary $\operatorname{Bd} A$ ? We will have a positive answer for it with the 0-dimensional exceptions. Those exceptions are easy to treat, which we call the trivial exceptions. Beside the trivial exceptions, there is a simple method constructing $A$ from a given $\Omega$ by filling "outside" of $\Omega$. We call the topology of such an $A$ the basic type. Conversely, for a prescribed attractor $A$, is $\operatorname{Bd} A$ an $\omega$-limit set? This question needs a more correct refinement, because $A$ may not have the connected $\operatorname{Bd} A$, and in the case, $\operatorname{Bd} A$ is never an $\omega$-limit set. Even in the case $\operatorname{Bd} A$ is connected, the question cannot be true. Therefore, we ask if an attractor $A$ is of the basic type, $\mathrm{Bd} A$ is an $\omega$-limit set. We will give a positive answer in $\S$. For any attractor $A$, it is a finite intersection of basic types and thus $\mathrm{Bd} A$ is a finite union of $\omega$-limit sets.

Our result should be compared with Jiménez López and Peralta-Salas' work [10]. Their result characterizes every possible topology of "global attractors" of local real analytic attractors. The global attractor $G$ contains finitely many compact attractors (used in our paper) and all the $\omega$-limit sets (see the precise definition in [10, pp.3]). Therefore, the global attractor should reflect the simultaneous orbits' behavior in itself. But, it cannot exist in many cases of local real analytic flows and polynomial flows. (The main theorem of Jiménez López and Peralta-Salas tells the topology of the global attractors, if it exists.) For instance, many polynomial flows (in fact most of them) have an orbit with the $\omega$-limit set homeomorphic to $\mathbb{R}^{1}$.

The attractor in our paper, in contrast, does not assume the compactness. It seemingly reflects an $\omega$-limit set of only one orbit. However, since any attractor is a finite intersection of attractors of the basic type, our attractor sufficiently reflects the $\omega$-limit sets whichever we want to look at. Eventually, some attractor separates $\mathbb{R}^{2}$ into $R_{1}, \ldots, R_{l}$ so that each region $R_{i}$ is simply connected and admits a global attractor, denoted by $G_{i}$. Now the method of the proof in [10] is applicable to the respective global attractor $G_{i}$ and one can see that $G_{i}$ is a "flower" in the language of $[10]$.

The attractor is defined after Conley [6] with a slight modification followed by 
later works, as below. (See also [5],[12],[11]).

Definition 1.1. Let $\Phi$ be a local real analytic flow by $z^{\prime}=f(z)$. An attractor $A$ is a closed proper nonempty subset of $\mathbb{R}^{2}$ invariant under $\Phi$ (i.e. for every $x \in A$, $Z_{x}(t) \in A$ for all $t \in\left[0, b_{x}\right)$ ), such that there exists an open neighborhood $U$ of $A$ satisfying

$$
\bigcap_{t \geq 0} \Phi(t, U)=A
$$

The above definition means that $x \in \bigcap_{t \geq 0} \Phi(t, U)$ if and only if $Z_{x}(s) \in U$ for all $s \in\left(a_{x}, 0\right]$. One needs to be careful when saying $\Phi(t, B)$ for a subset $B \in \mathbb{R}^{2}$, because the integral curves may not be complete. We say $x \in \Phi(t, B)$ if there exists $y \in B$ such that $t \in\left(a_{y}, b_{y}\right)$ and $\Phi(t, y)=x$, equivalently, $-t \in\left(a_{x}, b_{x}\right)$ and $\Phi(-t, x) \in B$. In fact, we can avoid the iterated use of $a_{x}, b_{x}, a_{y}, b_{y}, \ldots$ in many statements by globalizing local flows (see the discussion of Remark 2.3).

Recently, Jiménez López-Llibre [9] characterized the $\omega$-limit sets for analytic flows on the plane (up to homeomorphisms).

Theorem 1.2. ([9, Theorem A]) Let $\Phi$ be a local real analytic flow by $z^{\prime}=f(z)$ on $\mathbb{R}^{2}$. Let $\Omega=\omega_{\Phi}(u)$ for some $u \in \mathbb{R}^{2}$. Then $\Omega$ is one of the following:

(a) the empty set;

(b) a single point;

(c) the boundary of a cactus;

(d) the union of a circle $C$ and the boundaries of finitely many piecewise disjoint cacti, each of them contained in the disk enclosed by $C$ and intersecting $C$ at exactly one point;

(e) a union of the boundaries of countably many cacti, half-planes and chains, which are pairwise disjoint except that each cactus intersects either one of the halfplanes or one of the chains at exactly one point; moreover, every bounded set of $\mathbb{R}^{2}$ intersects finitely many of these sets.

A cactus is a simply connected finite union of homeomorphic closed discs where any two disks have at most one common point. A subset $A$ of $\mathbb{R}^{2}$ calls a half-plane if both $A$ and $\mathbb{R}^{2}-\operatorname{int}(A)$ are homeomorphic to $\left\{(x, y) \in \mathbb{R}^{2}: x \geq 0\right\}$. We also define that $A \subseteq \mathbb{R}^{2}$ is a chain if there exist disks $\left\{D_{i}\right\}_{i=1}^{\infty}$ such that:

(1) $A=\cup_{i=1}^{\infty} D_{i}$;

(2) if $|i-j|=1$, then $D_{i} \cap D_{j}$ consists of exactly one point; otherwise $D_{i} \cap D_{j}=\emptyset$;

(3) the disks $D_{i}$ tend to $\infty$; in other words, every bounded set of $R^{2}$ intersects finitely many disks $D_{i}$.

Their work, hence, motivates the concrete description of attractors in accordance with the explicit $\omega$-limit sets. Our result is the following.

Theorem 1.3.([3, Theorem A]) Let $\Phi$ be a local real analytic flow by $z^{\prime}=f(z)$ on $\mathbb{R}^{2}$. Suppose the $\omega$-limit set $\Omega$ of $u_{0} \in \mathbb{R}^{2}$ (with respect to $\Phi$ ) is a 1-dimensional set. 
Then, there exists an attractor $A$ such that the boundary $\mathrm{Bd} A=\Omega$. Furthermore, the attractor $A$ is homeomorphic to one of the following

(a) a cactus;

(b) the union of pairwise disjoint cacti, and a complement of a closed disk $B$ such that $C=\mathrm{Bd} B$ encloses all the cacti and each cactus intersects $C$ exactly by one point;

(c) a union of countably many cacti, half-planes and chains, which are pairwise disjoint except that each cactus intersects either one of the half-planes or one of the chains at exactly one point; moreover, every bounded set of $\mathbb{R}^{2}$ intersects finitely many of these sets.

Theorem 1.3 and its proof are based upon Jiménez López-Llibre's theorem ([9, Theorem A]) (principally the local analysis through the desingularization technique therein). In [9], Jiménez López and Llibre obtained more extensive results for the polynomial flows and the analytic flows on the sphere and the real projective plane, with more restrictive conclusions. In fact, their results include the converse statements, i.e., any subset of the type in the list of Theorem 1.2, is always expressed as the $\omega$-limit set of a local real analytic flow (see the statements of the theorems in $[9, \S 2]$ and $[9, \S 6])$. Hence, all the cases in our theorem are not vacuous ones.

The study of the behavior of a local real analytic flow along the boundary of the attractors, is our second aim.

We define the basin of attraction of $A$, denoted by $B(A)$, as

$$
\{q \in M \mid \omega(q) \neq \emptyset, \omega(q) \subseteq A\} .
$$

\section{The $\omega$-limit Sets for Analytic Flows}

In this section, we are going to prove Theorem 2.5. Before the proof, we need to study some general aspect of local flows and attractors. Let $\Phi$ be a local flow by an ODE $\left(x_{1}(t)^{\prime}, \cdots, x_{n}(t)^{\prime}\right)=g\left(x_{1}(t), \cdots, x_{n}(t)\right)\left(t \in \mathbb{R}^{1}\right)$ where $g: \mathbb{R}^{n} \rightarrow \mathbb{R}^{n}$ is a continuous function $(n \geq 1)$. We denote by $\left(a_{x}, b_{x}\right)$ the maximal domain where the ODE is solvable with the initial condition $x=\left(x_{1}(0), \cdots, x_{n}(0)\right)$. As in Definition 1.1 , a proper closed $\Phi$-invariant subset $A$ of $\mathbb{R}^{n}$ is called an attractor if there is an open neighborhood $U$ of $A$ such that $A=\bigcap_{t>0} \Phi(t, U)$. The meaning of the intersection was explained after Definition 1.1 for $n=2$, and in our case, it is adopted in a direct way. For a given attractor $A$, the associated open neighborhood $U$ is called an attractor block. The basin $B(A)$ of $A$, as in the case of $n=2$, is the set of $u \in \mathbb{R}^{n}$ satisfying $\omega(u)$ is a nonempty subset of $A$.

In this general situation, we will have two descriptions on the attractors and the orbits in Propositions 2.1 and 2.4.

Lemma 2.1. Let $\Phi$ be a local flow. Let $A$ be an attractor and $B(A)$ be a basin of attractor. If a point $x$ is in $B(A)-A$, then the intersection of the (positive) $\Phi$-orbit of $x$ and the attractor is empty.

Proof. Let $O_{\Phi}^{+}(x)$ be the (positive) $\Phi$-orbit of $x$, i.e.

$$
O_{\Phi}^{+}(x)=\left\{\Phi(t, x) \mid t \in\left[0, b_{x}\right)\right\} .
$$


Suppose that $O_{\Phi}^{+}(x) \cap A \neq \emptyset$. Thus there exists $t_{0} \geq 0$ such that $\Phi\left(t_{0}, x\right) \in A$ where $U$ is an attractor block for the attractor $A$. Therefore, denoting $x_{0}=\Phi\left(t_{0}, x\right)$, we have $x_{0}=\Phi\left(t_{0}, x\right) \in \bigcap_{t \geq 0} \Phi(t, U)$ So for all $t \geq 0$,

$$
x=\Phi\left(-t_{0}, x_{0}\right) \in \Phi\left(-t_{0}, \Phi(t, U)\right)=\Phi\left(-t_{0}+t, U\right) .
$$

Since $\bigcap_{t \geq-t_{0}} \Phi(t, U) \subseteq \bigcap_{t \geq 0} \Phi(t, U), x \in \bigcap_{t \geq 0} \Phi(t, U)$, i.e. $x \in A$. This is a contradiction, so the intersection is empty.

Remark 2.2. One obvious remark is that even if, in the definition of attractors, we drop the condition " $A$ is $\Phi$-invariant", $A$ is necessarily $\Phi$. This is because $A=\bigcap_{t>0} \Phi(t, U)$ for some open neighborhood $U$ of $A$.

Remark 2.3. For a given local flow $\Phi$ on $\mathbb{R}^{n}$ by a continuous function $g$, we can normalize $\Phi$ in a certain sense so that the normalized flow $\Phi^{\prime}$ is a (global) flow, i.e. every integral curve of $\Phi^{\prime}$ is complete and its trajectory coincides the integral curve of $\Phi$. Indeed, by replacing $g$ to a bounded continuous function $g^{\prime}$ (after a suitable rescaling), we have the induced flow $\Phi^{\prime}$ satisfying those properties. This is well-known procedure in which a local flow $\Phi$ is topologically equivalent to a flow $\Phi^{\prime}$. Since $\Phi^{\prime}$ is a flow (in the ordinary sense), $\Phi^{\prime}(t, B)$ for any $t \in \mathbb{R}^{1}$ and any subset $B \in \mathbb{R}^{n}$ is well-defined, but $\Phi^{\prime}(t, B) \neq \Phi(t, B)$ in general. In the case $\Phi$ is a local real analytic flow, we cannot expect $\Phi^{\prime}$ is a real analytic flow. However, $A \subset \mathbb{R}^{n}$ (resp. $\Omega$ ) is an attractor (resp. $\omega$-limit set) of $\Phi$ if and only if it is an attractor (resp. $\omega$-limit set) of $\Phi^{\prime}$. Of course, in some arguments, we could save the labors, avoiding the iterated use of $a_{x}, b_{x}$, etc.

We can say further: the $\Phi$-invariance of the boundaries of the attractors.

Proposition 2.4. Let $A$ be an attractor of a local flow $\Phi$. Then $\Phi(\operatorname{Bd} A, t) \subset \operatorname{Bd} A$ for all $t \geq 0$, i.e., the boundary of an attractor for a local flow is positive invariant for the flow.

Proof. Suppose the contrary of the conclusion. Then by the invariance of attractors, we may assume that there exist $x \in \operatorname{Bd} A$ and $t_{0}>0$ such that $\Phi\left(t_{0}, x\right)$ is in the interior of $A$. From the local compactness, we can choose a compact neighborhood $C$ of $\Phi\left(t_{0}, x\right)$ in the interior of $A$ such that $\Phi\left(-t_{0}, C\right)$ is also a compact neighborhood of $x$. Thus, we can pick $y \in U-A$ where $U$ is an attractor block of $A$ such that $\Phi\left(t_{0}, y\right) \in A$. This contradicts Lemma 2.1. Hence the boundary is positive invariant for the analytic flow.

Theorem 2.5. Let $\Phi$ be a local real analytic flow by $z^{\prime}=f(z)$ on $\mathbb{R}^{2}$. Let $A$ be a nonempty attractor with dimension greater than 1 and $B(A)$ be the basin of attractor. Assume that $\operatorname{dim}(\operatorname{Bd} A \cap \operatorname{Sing} \Phi)=0$. Then, for every $u \in B(A)-A$, the $\omega$-limit set $\omega(u)$ is in the boundary of A and homeomorphic to one of (b)-(e) in Theorem 1.2.

Proof. Let $u \in B(A)-A$. By the definition of a basin of attractor, the $\omega$-limit set of $u$ is nonempty and is contained in the attractor $A$. Using Lemma 2.1, we 
obtain that $\omega(u) \subseteq \operatorname{Bd} A$. Indeed, assume that $\omega(u) \nsubseteq \operatorname{Bd} A$. Then we get that $\omega(u) \cap(B(A)-A) \neq \emptyset$ which is a contradiction for the definition of a basin of attraction.

Next, we suppose that the boundary of the attractor is smooth for $\Phi$. So we can say that the $\omega$-limit set $\omega(u)$ is a subset of one of the sets described in (c)-(e) in Theorem 1.2. Otherwise, by the hypothesis $\operatorname{dim}(\operatorname{Bd} A \cap \operatorname{Sing} \Phi)=0$, the $\omega$-limit set is a one-point set in $\operatorname{Bd} A$.

Remark 2.6. The problem for the description of an $\omega$-limit set for a flow is so interesting and important to study dynamical systems. Given an attractor, the shape of the $\omega$-limit set from the basin of the attractor is completely dependent upon the characterization of the attractor.

Corollary 2.7. Let $\Phi$ be a local real analytic flow by $z^{\prime}=f(z)$ on $\mathbb{R}^{2}$ and $A$ a nonempty compact attractor with dimension greater than 1 . Assume that $\operatorname{dim}(\operatorname{Bd} A \cap \operatorname{Sing} \Phi)=0$. Then the $\omega$-limit set from the basin of $A$ is compact and homeomorphic to one of (b)-(d) in Theorem 1.2.

Remark 2.8. The problem for the description of an $\omega$-limit set for a flow is so interesting and important to study dynamical systems. Given an attractor, actually, the shape of the $\omega$-limit set from the basin of the attractor is completely dependent upon the characterization of the attractor.

\section{References}

[1] A. A. Andronov, E. A. Leontovich, I. I. Gordon and A. G. Măier, Qualitative theory of second-order dynamic systems, Translated from the Russian by D. Louvish. Halsted Press (A division of John Wiley \& Sons), New York-Toronto, Ont., Jerusalem-London, 1973.

[2] V. I. Arnol'd and Y. S. Il'yashenko, Ordinary differential equations, [Current problems in mathematics. Fundamental directions, Vol. 1, 7-149, Akad. Nauk SSSR, Vsesoyuz. Inst. Nauchn. i Tekhn. Inform., Moscow, 1985; MR0823489 (87e:34049)]. Translated from the Russian by E. R. Dawson and D. O'Shea. Encyclopaedia Math. Sci., 1, Dynamical systems, I, 1-148, Springer, Berlin, 1988.

[3] J. Choy and H.-Y. Chu, Attractors on Riemann spheres, submitted.

[4] J. Choy and H.-Y. Chu, On the Envelopes of Homotopies, Kyungpook Math. J., 49(3)(2009), 573-582

[5] C. M. Carballo and C. A. Morales, Omega-limit sets close to singular-hyperbolic attractors, Illinois J. Math., 48(2004), 645-663.

[6] C. Conley, Isolated invariant sets and the morse index, C. B. M. S. Regional Lect., 38, A. M. S., 1978

[7] F. Dumortier, Singularities of vector fields on the plane, J. Differential Equations, 23(1977), 53-106. 
[8] F. Rodriguez Hertz and J. Rodriguez Hertz, Expansive attractors on surfaces, Ergodic Theory Dynam. Systems, 26(2006), 291-302.

[9] V. Jiménez López and J. Llibre, A topological characterization of the $\omega$-limit sets for analytic flows on the plane, the sphere and the projective plane, Adv. Math., 216(2007), 677-710.

[10] V. Jimenez Lopez and D. Peralta-Salas, Global attractors of analytic plane flows, To appear in Ergodic Theory Dynam. Systems.

[11] C. A. Morales, M. J. Pacifico and E. R. Pujals, Robust transitive singular sets for 3-flows are partially hyperbolic attractors or repellers, Ann. of Math., 160(2004), 375-432.

[12] J. Milnor, On the concept of attractor, Commun. Math. Phy., 99(1978), 177-195.

[13] J. C. Robinson and O. M. Tearne, Boundaries of attractors of omega limit sets, Stoch. Dyn., 5(2005), 97-109.

[14] A. Seidenberg, Reduction of singularities of the differential equation $A d y=B d x$. Amer. J. Math., 90(1968), 248-269. 